\title{
Correction: Voluntary and spontaneous facial mimicry toward other's emotional expression in patients with Parkinson's disease
}

\author{
June Kang, Dilara Derva, Do-Young Kwon, Christian Wallraven
}

In the Stimulus subsection of the Materials and methods section, there is an incorrect reference in the third sentence.

The correct sentence is: Expression stimuli were chosen from the East-Asian Dynamic Facial Expression Stimulus (EADFES) database (Kang et al., 2015), which contains stimuli created and verified based on suggested action units related to each emotion (Anger: 4CDE $+5 \mathrm{CED}+7+17+23 / 24$, Joy: $6+12 \mathrm{CDE}+25$, Sad: $1+4+15 \mathrm{ABC}+17$, [38]).

The reference is: Kang J, Ham BJ. Development and Validation of East-Asian Dynamic Facial Expression Stimulus. Proceedings of Current Trends in Biomedical Science. 2015. Seoul, South Korea.

\section{Reference}

1. Kang J, Derva D, Kwon D-Y, Wallraven C (2019) Voluntary and spontaneous facial mimicry toward other's emotional expression in patients with Parkinson's disease. PLoS ONE 14(4): e0214957. https:// doi.org/10.1371/journal.pone.0214957 PMID: 30973893

\section{G OPEN ACCESS}

Citation: Kang J, Derva D, Kwon D-Y, Wallraven C (2019) Correction: Voluntary and spontaneous facial mimicry toward other's emotional expression in patients with Parkinson's disease. PLOS ONE 14 (5): e0217715. https://doi.org/10.1371/journal. pone.0217715

Published: May 28, 2019

Copyright: @ 2019 Kang et al. This is an open access article distributed under the terms of the Creative Commons Attribution License, which permits unrestricted use, distribution, and reproduction in any medium, provided the original author and source are credited. 